International Journal of Pure and Applied Mathematics

Volume 114 No. $4 \quad 2017,709-717$

ISSN: 1311-8080 (printed version); ISSN: 1314-3395 (on-line version)

url: http://www.ijpam.eu

doi: 10.12732 /ijpam.v114i4.2

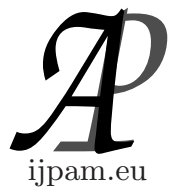

\title{
FILTERS IN SEMITRELLISES
}

\author{
Shashirekha B. Rai ${ }^{1}$, Prashantha Rao ${ }^{2 \S}$ \\ ${ }^{1}$ Depatrment of Mathematics \\ NMAMIT Nitte (Affiliated to VTU) \\ Karkal Taluk, Karnataka State, INDIA \\ ${ }^{2}$ Department of Mathematics \\ Vivekananda College of Engineering and Technology (Affiliated to VTU) \\ Nehru Nagar, Puttur, 574203, Karnataka State, INDIA
}

\begin{abstract}
In this paper we have introduced the concept of semitrellises as a generalization of semilattices. Some characterizations of filters in semitrellises are obtained. Also we have proved that a dense subset $\mathrm{D}$ of a semitrllis $\mathrm{S}$ is an upset if $\mathrm{D}$ is meet associative.
\end{abstract}

AMS Subject Classification: 06B20, 06B10

Key Words: poset, lattice, psoset, semitrellis, filter

\section{Introduction}

A reflexive and antisymmetric binary relation $\unlhd$ on a set $A$ is called a pseudoorder on $\mathrm{A}$ and $\langle A, \unlhd\rangle$ is called a pseudo-ordered set or a psoset. For $a, b \in A$ if $a \unlhd b$ and $a \neq b$, then we write $a \triangleleft b$. For a subset $B$ of $A$, the notions of a lower bound, an upper bound, the greatest lower bound (meet, denoted by $\wedge B$ ), the least upper bound (join, denoted by $\vee B$ ) are defined analogous to the corresponding notions in a poset (refer[2]).

It is shown in [5] that any psoset can be regarded as a digraph(possibly infinite) in which for any pair of distinct elements $u$ and $v$ there is no directed line between $u$ and $v$ or if there is a directed line from $u$ to $v$, there is no directed line from $v$ to $u$.

Received: June 30, 2016

Revised: $\quad$ May 22, 2017

Published: June 7, 2017

$\S$ Correspondence author (c) 2017 Academic Publications, Ltd. url: www.acadpubl.eu 
A psoset $\langle A, \unlhd\rangle$ in which every pair of elements has a least upper bound and a greatest lower bound is called a trellis. In other words, a trellis is an algebra $\langle L, \wedge, \vee\rangle$ where the binary operations $\wedge$ and $\vee$ satisfy the following properties:

(i) $a \vee b=b \vee a$ and $a \wedge b=b \wedge a$ for all $a, b \in L$;

(ii) $a \vee(b \wedge a)=a=a \wedge(b \vee a)$ for all $a, b \in L$;

(iii) $a \vee((a \wedge b) \vee(a \wedge c))=a=a \wedge((a \vee b) \wedge(a \vee c))$ for all $a, b, c \in L$.

The notion of a trellis as a non-associative generalisation of a lattice is due to E.Fried [1] and H.L.Skala [6]. A psoset $S$ is said to be a meet semitrellis if for all $a, b \in S, a \wedge b \in S$. Dually we can define a join semitrellis. In this paper by a semitrellis we mean a meet semitrellis.

\section{2. definitions and Results}

Definition 2.1. A meet semitrellis is called directed above if for all $a, b \in$ $S$, there exists an element $c \in S$ such that $c \unrhd a$ and $c \unrhd b$.

Meet semitrellis $S$ directed above will be denoted by $S^{\Delta}$ for all future references.

Definition 2.2. A nonempty subset $I$ of $S^{\Delta}$ is called a downset if for $x \in I$ and $y \in S, y \unlhd x$ imply $y \in I$. Downset is also called an ideal if for $x, y \in I, x \vee y \in I$

Definition 2.3. A nonempty set $F$ of $S^{\Delta}$ is called an upset if for $x \in F$ and $y \in S, y \unrhd x$ imply $y \in F$. An upset $F$ of $S$ is also called a filter if for all $x, y \in F, x \wedge y \in F$.

Remark 2.4. Every ideal is a downset as well as an upset but not the converse

Definition 2.5. An ideal (downset) $P$ of $S^{\Delta}$ is called a prime ideal (downset) if $a \wedge b \in P$ imply either $a \in P$ or $b \in P$.

Definition 2.6. A filter $F$ of $S^{\Delta}$ is called prime if $S-F$ is a prime ideal.

Definition 2.7. A filter $F$ of $S^{\Delta}$ is called a maximal filter if $F \neq S$ and $F$ is not contained in any other proper filter of $S^{\Delta}$.

Definition 2.8. A prime downset is called a minimal prime downset if it does not contain any other prime downset of $S$.

Definition 2.9. In $S^{\Delta}$, an ideal $J$ is called semiprime ideal if for $x, y, z \in$ $S, x \wedge y \in J$ and $x \wedge z \in J$ imply that $x \wedge(y \vee z) \in J$. 
Theorem 2.10. Let $F$ be a nonempty proper subset of a semitrellis $S$. then $F$ is a filter if and only if $S-F$ is a prime downset.

Proof. Let $F$ be a filter of a semitrellis $S$. Let $x \in S-F$ and $y \unlhd x$. Then clearly $y \notin F$. Theerfore $y \in S-F$ and $S-F$ becomes a proper downset.

Claim: $S-F$ is a prime downset.

Let $a, b \in S$ such that $a \wedge b \in S-F$. Then $a \wedge b \notin F$ and hence either $a \notin F$ or $b \notin F$ as $F$ is a filter. Therefore either $a \in S-F$ or $b \in S-F$ and hence $S-F$ is a prime downset.

Conversely, let $S-F$ be a prime downset. For $x, y \in F$ we have $x \wedge y \notin S-F$. Therefore $x \wedge y \in F$. Suppose $x \in F$ and $y \unrhd x$. Then $x \notin S-F$ and hence $y \notin S-F$. This implies $y \in F$, proving that $F$ is a filter.

Theorem 2.11. Let $F$ be a nonempty subset of a semitrellis $S$.Then $F$ is a maximal filter if and only if $S-F$ is a minimal prime downset.

Proof. Let $F$ be a maximal filter of $S$. If $S-F$ is not a minimal prime downset, then there exists a prime downset $I \subseteq S-F$. This implies $F \subseteq S-I$, contradicting the maximality of $F$.

Conversely, let $S-F$ be a minimal prime downset. If $F$ is not a maximal filter, then there exists a proper filter $G$ of $S$ such that $F \subseteq G$. Then $S-G \subseteq$ $S-F$, contradicting the minimality of $S-F$.

Definition 2.12. For a subset $A$ of a psoset $P$ the set

$$
A^{\mathrm{u}}=\{x \in P: x \unrhd a \forall a \in A\}
$$

is called the upper cone of $A$.

Lower cone $A^{\mathrm{l}}$ of $A$ is the dual notion of $A^{\mathrm{u}}$.

Definition 2.13. An element $d$ of a trellis $L$ is said to be:

(i) right transitive if $d \unlhd x \unlhd y$ imply $d \unlhd y$;

(ii) left transitive if $x \unlhd y \unlhd d$ imply $x \unlhd d$;

(iii) middle transitive if $x \unlhd d \unlhd y$ imply $x \unlhd y$.

Definition 2.14. A subset $I$ of a psoset $P$ is called an ideal if $a, b \in I$ implies $(a, b)^{\mathrm{ul}} \subseteq I$.

A proper ideal $I$ is called prime if $(a, b)^{l} \subseteq I$ imply either $a \in I$ or $b \in I$.

Filter and prime filter can be defined dually.

Given a left transitive element $a$ of a psoset $P$, the subset $\{x \in P: x \unlhd a\}$ is an ideal, called principal ideal generated by $a$ and is denoted by $(a]$. Dually we have the definition of principal filter. 
Definition 2.15. An ideal $I$ of a psoset $P$ is called semiprime if $(a, b)^{\mid} \subseteq I$ and $(a, c)^{\mathrm{l}} \subseteq I$ together imply that $\left\{a,(b, c)^{\mathrm{u}}\right\}^{\mathrm{I}} \subseteq I$

Lemma 2.16. Let $I$ be an ideal of a psoset $P$. If $I$ is prime, then $I$ is semiprime.

Proof. Let $I$ be a prime ideal of a psoset $P$ and for $a, b, c \in P$ let $(a, b)^{1} \subseteq I$ and $(a, c)^{\mid} \subseteq I$. Since $I$ is prime, we have:

Case (i): If $a \in I$, then $\left\{a,(b, c)^{\mathrm{u}}\right\}^{\mathrm{l}} \subseteq a^{\mathrm{l}} \subseteq I$.

Case (ii): If $a \notin I$, then $b, c \in I$ and hence $(b, c)^{\mathrm{ul}} \subseteq I$ Therefore $\left\{a,(b, c)^{\mathrm{u}}\right\}^{\mathrm{l}} \subseteq$ $(b, c)^{\mathrm{ul}} \subseteq I$. Thus $I$ is semiprime.

Remark 2.17. Converse of lemma 2.17 need not be true. In the psoset of Figure 1, $I=\{c, d\}$ is semiprime ideal which is not prime.

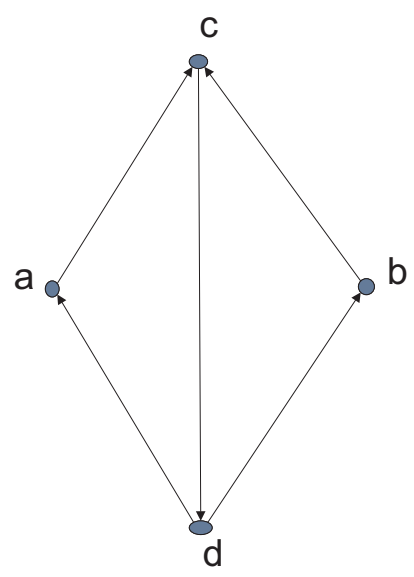

Figure 1

Definition 2.18. An element $x$ of a psoset is meet irreducible if $x$ can not be obtained as a meet of two elements different from $x$.

Theorem 2.19. Every prime ideal of a psoset $P$ is a meet irreducible element of $\operatorname{Id}(P)$

Proof. Let $I$ be a prime ideal of $P$ such that $I=J \cap K$ for $J, K \in I d(P)$. We need to prove that either $I=J$ or $I=K$. Clearly $I \subseteq J$ and $I \subseteq K$. Suppose $I \neq J$ and $I \neq K$, then there exists elements $x, y \in P$ such that $x \in J-I$ and $y \in K-I$. But as $J$ and $K$ are ideals, we must have $(x, y)^{\prime} \subseteq J \cap K=I$ and $I$ is a prime ideal, so either $x \in I$ or $y \in I$, a contradiction to the fact that $x, y \notin I$. 


\section{Weakly Distributive Semitrellises and * Semitrellises}

Definition 3.1. A semitrellis $S$ with least element 0 is called weaklydistributive semitrellis if for any $a, b, c \in S$ such that $a \wedge b=0=a \wedge c$ implies $a \wedge d=0$ for some $d \unrhd b, c$.

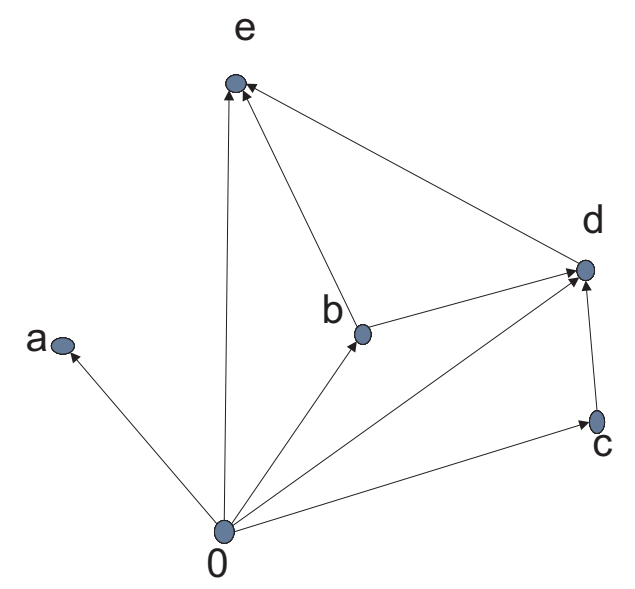

Figure 2

The semitrellis in Figure 2 is an example for a weakly distributive semitrellis.

Lemma 3.2. Let $S$ be a directed above semitrellis with 0 . If $S$ is not weakly distributive, then the set of all middle transitive elements $F=\{x \in$ $S \mid x \unrhd a \wedge y \neq 0 \quad \forall y \unrhd b, c\}$ where $a, b, c \in S$ such that $a \wedge b=a \wedge c=0$ is a proper filter.

Proof. If $S$ is not weakly distributive, there are elements $p, q, r \in S$ such that $p \wedge q=p \wedge r=0$ and $p \wedge d \neq 0$ for all $d \unrhd q$, $r$. As $p \unrhd p \wedge d$, we have $p \in F$. Hence $F$ is non empty. Clearly $0 \notin F$. We prove that $F$ is a filter. Let $x \in F$ and $z \unrhd x$. Then $x \unrhd a \wedge y$ for all $y \unrhd b, c$ so that $a \wedge y \unlhd x \unlhd z$. Since $x$ is middle transitive $z \unrhd a \wedge y$. Hence $z \in F$.

Let $x, z \in F$. Then $x \unrhd a \wedge y$ and $z \unrhd a \wedge y$ for all $y \unrhd b, c$. Then $x \wedge z \unrhd a \wedge y$ for all $y \unrhd b, c$ so that $x \wedge z \in F$ Thus $F$ is a filter.

The following definition of weakly distributive semitrellis is equivalent to definition 3.1 .

Definition 3.3. A semitrellis $S$ with 0 is said to be a weakly distributive 
semitrellis if $(a)^{*}$ is an ideal of $S$ for any $a \in S$, where

$$
(a)^{*}=\{x \in S / x \wedge a=0, a \in S\}
$$

Definition 3.4. If $S$ is a semitrellis with 0 , then for any subset $A$ of $S$, we define $A^{*}=\{x \in S / x \wedge a=0, \forall a \in A\}$

Remark 3.5. If $A=\{a\}$ then $A^{*}=(a)^{*}$

Definition 3.6. A semitrellis $S$ with 0 is said to be a ${ }^{*}$ semitrellis if and only if for any $a \in S$ there exists $a^{1} \in S$ such that $(a)^{*}=\left(a^{1}\right)^{* *}$ where $a \wedge a^{1}=0$

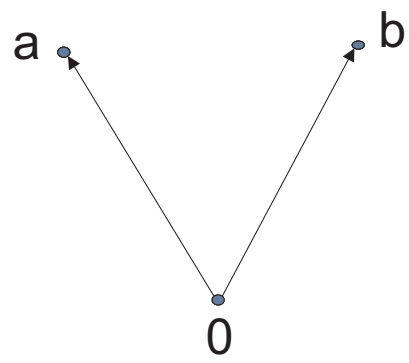

Figure 3

Semitrellis of Figure 3 is weakly distributive as well as ${ }^{*}$ semitrellis.

Definition 3.7. An element $a \in S$ is called a dense element of $S$ if and only if $(a)^{*}=\{0\}$

Definition 3.8. A subset $D$ of a semitrellis $S$ is called dense if all the elements in $D$ are dense elements.

Definition 3.9. An element $x$ of a semitrellis $S$ is called meet associative if $x \wedge(y \wedge z)=(x \wedge y) \wedge z$ for all $y, z \in S$

Definition 3.10. A subset $D$ of a semitrellis $S$ is said to be meet associative if every element of $D$ are meet associative.

Proposition 3.11. A dense subset $D$ of a semitrellis $S$ is an upset if $D$ is meet associative.

Proof. Let $D$ be a meet associative dense subset of $S$. If $x \in D$ and $a \in S$ such that $a \unrhd x$. If $a \notin D$, then there exists an element $y \in S$ such that $a \wedge y=0$. Consider $x \wedge y=(x \wedge a) \wedge y=x \wedge(a \wedge y)=0$ which is a contradiction to the fact that $x$ is a dense element. Therefore $a \in D$ and hence $D$ is an upset. 
Remark 3.12. Meet associativity in proposition 3.11 is only a sufficient condition but not necessary. Consider the semitrellis in the in Figure 4. Here if $D=\{a, b, c\}$, thrn $D$ is an upset but $D$ is not meet associative.

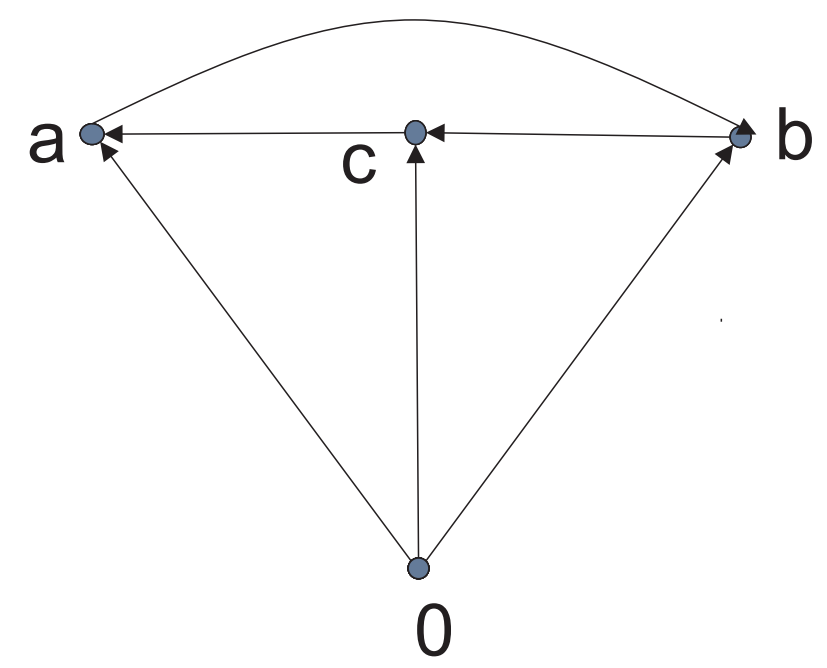

Figure 4

Theorem 3.13. In a weakly distributive semitrellis $S$, the following are equivalent.

(i) $S$ is a * semitrellis;

(ii) For any $x \in S$, there exists $x^{1} \in S$ such that $x \wedge x^{1}=0$ and $[x) \cap\left[x^{1}\right) \subseteq D$ where $D$ is a dense filter.

Proof. Let $S$ be a weakly distributive semitrellis, then for every $a \in S,(a)^{*}=$ $\{x \in S / x \wedge a=0\}$ is an ideal.

$(i) \Rightarrow(i i)$ is obvious.

$($ ii $) \Rightarrow(i)$ Clearly $\left(x^{1}\right)^{* *} \subseteq(x)^{*}$ because if $s \in\left(x^{1}\right)^{* *}$ then $s \wedge r=0$ for all $r \in\left(x^{1}\right)^{*}$. Therefore $s \wedge x=0$ as $x \in\left(x^{1}\right)^{*}$ and hence $s \in(x)^{*}$.

Also let $t \in s$ be such that $t \wedge x=0$ and $r \in S$ be such that $r \wedge x^{1}=0$. Then as $x$ is meet associative $x \wedge(r \wedge t)=(r \wedge t) \wedge x=r \wedge(t \wedge x)=r \wedge 0=0$ which imply $x \in(r \wedge t)^{*}$. Similarly we can prove that $x^{1} \in(r \wedge t)^{*}$. Since for $t, r \in S$, we have $t \wedge r \in S$ and $t \wedge r)^{*}$ is an ideal of $S$ it follows that there exists an element $z \in S$ such that $z \unrhd x, x^{1}$ and $z \in(t \wedge r)^{*}$ which imply $z \wedge(t \wedge r)=0$. Since $z \unrhd x, x 1$, we have $z \in[x)$ and $z \in\left[x^{1}\right)$ so that $z \in[x] \cap\left[x^{1}\right) \subseteq D$. Therefore $z \in D$ and hence $t \wedge r=0$. Thus $t \in(r)^{*}$. But $r \in\left(x^{1}\right)^{*}$ and $t \wedge r=0$ 
implies that $t \in\left(x^{1}\right)^{* *}$ so that $(x)^{*} \subseteq\left(x^{1}\right)^{* *}$. Therefore $(x)^{*}=\left(x^{1}\right)^{* *}$ proving that $S$ is a ${ }^{*}$ semitrellis.

\section{Nodal Filters in Semitrellises}

In [7], the concept of nodal elements is introduced in trellises and its characterizations are obtained. In this section we obtain properties of nodal filters in semitrellises.

Let $F(S)$ denotes the set of all filters of an updirected semitrellis $S$. When ordered by set inclusion $F(S)$ is a lattice in which for every $F, G \in F(S), F \wedge G=$ $F \cap G$ and $F \vee G$ is the filter generated by $F \cup G$. That is $F \vee G=[F \cup G)$.

Definition 4.1. A node in a poset $P$ is an element $a$ which is comparable with every element of $P$

Definition 4.2. A node in a psoset $P$ is an element $a$ which is middle transitive and comparable with every element of $P$.

Theorem 4.3. For a filter $F$ of a semitrellis $S$, the following conditions are equivalent:

(i) the relation $x \triangleright y$ is satisfied for right transitive elements $x \in F$ and $y \in S-F$;

(ii) any filter $G$ of $S$ is comparable with $F$;

(iii) $F$ is a node of $F(S)$ where $F(S)$ is set of all filters of $F$.

Proof. $(i) \Rightarrow($ ii $)$ : Let us assume that there exists a filter $G$ which is incomparable with $F$. Then there are elements $x$ and $y$ such that $x \in F-G$ and $y \in G-F$ and $x \not y$, a contradiction.

$($ ii $) \Rightarrow($ iii $)$ : Obvious.

$($ iii $) \Rightarrow(i)$ : If $F$ is a node in $F(S)$, then for every right transitive element $x \in F$ and for $y \notin F$, we have $[y) \supset F$ hence $[y) \supset F \subset[x] \Rightarrow x \triangleright y$.

A filter satisfying one of the conditions in Theorem 4.3 is called a nodal filter. Obviously semitrellis $S$ is an improper nodal filter .

Theorem 4.4. For a right transitive element $x,[x)$ is nodal filter if and only if $x$ is a node in $S$.

Proof. Follows easily. 
Let $N(S)$ be the set of all nodal filters of a semitrellis $S$, ordered by set inclusion. Then $N(S)$ is a chain with the greatest element $S$. If $1 \in S$ then $N(S)$ has the least element [1).

Definition 4.5. Two elements $x$ and $y$ of a semitrellis $S$ are said to be connected ( ie $(x, y) \in R$ ) if there is no nodal filter separating $x$ and $y$.

Note that $(x, y) \notin R \Rightarrow$ either $x \triangleright y$ or $x \triangleleft y$

Theorem 4.6. In any semitrellis $S$, the relation $R$ satisfies the following properties.

1) $R$ is a congruence relation of $\langle S, \wedge\rangle$;

2) Any $R$-class contains at most one right transitive node.

Proof. (1) Clearly $(x, x) \in R$ for all $x \in S$. Also if $(x, y) \in R$ then $(y, x) \in R$ for all $x, y \in S$. Suppose $(x, y)$ and $(y, z)$ belong to $R$. If possible let $(x, z) \notin R$. Then there exists a nodal filter $F$ such that $x \in F$ and $z \notin F$. Then $y \in F$ and $(y, z) \notin R$ a contradiction. Therefore $(x, z) \in R$ and $R$ is an equivalence relation. Now if $(x, y) \in R$ then $(x \wedge s, y \wedge s) \in R$ for every $s \in S$. Otherwise, $x \wedge s \in F$ and $y \wedge s \notin F$ for some nodal filter $F$. Hence $x \in F, s \in F$ and $y \notin F$ which is a contradiction as $(x, y) \in R$. Therefore $R$ is a congruence relation in $\langle S, \wedge\rangle$.

(2) Let $a$ and $b$ be two right transitive connected nodes of $S$. Then we have either $a \triangleleft b$ or $b \triangleleft a$. If $a \triangleleft b$ then $a$ and $b$ are separated by a nodal filter $[b)$ which imply $(a, b) \notin R$, a contradiction.

\section{References}

[1] E.Fried, Tournaments and non associative lattices, Ann. Univ. Sci. Budapest Eotvos, Sect Math., 13 (1970), 151-164.

[2] G. Grätzer, General Lattice Theory, Second Edition, Birkhäuser Verlag, 2003.

[3] Mamtaz Begum, A.S.A. Noor, Semi prime ideals in semilattices, Annals of Pure and Applied Mathematics, 1, No. 2 (2012), 149-157.

[4] Mamtaz Begum, A.S.A. Noor, Some properties of 0 distributive semilattices, Annals of Pure and Applied Mathematics, 2, No. 1 (2012), 60-66.

[5] S. Parameshwara Bhatta, H. Shashirekha, A characterization of completeness for trellises, Algebra Universalis, 44 (2000), 218-233.

[6] H.L. Skala, Trellis theoy, Algebra Univeralis, 1 (1971), 218-233.

[7] Shashirekha B. Rai, Prashantha Rao, On nodes in trellises, International Journal of Pure and Applied Mathematics, 105, No. 4 (2015), 647-652. 
